\title{
Gas-Granular Simulation Framework for Spacecraft Landing Plume-Surface Interaction and Debris Transport Analysis
}

\author{
Peter A. Liever ${ }^{1}$, Manuel P. Gale ${ }^{1}$, Ranjan S. Mehta ${ }^{1}$, and Jeffrey S. West ${ }^{2}$ \\ ${ }^{1}$ Jacobs Space Exploration Group \\ CFD Research Corporation, Huntsville, Alabama \\ peter.liever@cfdrc.com \\ ${ }^{2}$ NASA Marshall Space Flight Center, Huntsville, Alabama \\ jeffrey.s.west@nasa.gov
}

\begin{abstract}
The Gas-Granular Flow Solver (GGFS) multi-phase flow computational framework has been developed to enable simulations of particle flows of complex extra-terrestrial regolith materials. Particle flows of interest include the damage of unprepared spacecraft landing sites from rocket plume impingement on Moon, Mars, and asteroids. The flow solver implements an Eulerian-Eulerian two-fluid model with fluid representation of the gas phase and granular phase to avoid the need to model billions of particle interactions. The granular phase is modeled as an Eulerian fluid with constituent physics closure models derived from first-principle Discrete Element Model (DEM) particle interaction simulations that capture the complex, non-linear granular particle interaction effects. Granular phase constituent models have been developed and integrated that address the granular material mechanics complexities resulting from both, the irregular, jagged particle shapes and poly-disperse mixture effects encountered in extra-terrestrial regolith, with lunar regolith as the extreme. The GGFS capabilities are being integrated into a proven NASA plume-surface interaction and debris transport simulation framework featuring the Loci/CHEM CFD program and Debris Transport Analysis (DTA) post-processing tools for applications in robotic and human Moon and Mars lander development. Integration of the three simulation tool components, Loci/CHEM, GGFS, and DTA, into a coordinated simulation framework will enable time-accurate spacecraft landing simulations that account for the alteration of the landing surface through plume induced cratering and the resulting redirection of plume impingement flow and debris transport. Initial implementation of this simulation framework and application examples will be presented.
\end{abstract}

\section{INTRODUCTION}

The liberation of debris particles caused by rocket plume impingement flow during spacecraft propulsive landing on the unprepared regolith of Moon, Mars, and other celestial bodies poses a high risk for robotic and human exploration activities. Regolith debris is lofted from the surface by the plume impingement gas flow and accelerated to high velocities with kinetic energy levels that pose a threat to the vehicle itself and any nearby sensitive objects.

The progression of surface regolith liberation from dust lofting through viscous erosion to full bearing capacity failure with deep cratering has been observed for the Skycrane landing operation delivering the Opportunity Mars Science Lab (MSL). Severe erosion 
with soil bearing capacity failure began below 18 meter elevation before the Skycrane hovered. Craters were formed by the plumes impingement of the relatively small Skycrane engines (Vizcaino\&Mehta, 2015) even without an actual landing. Significant soil erosion levels and landing surface cratering will occur for future human exploration of Mars because of larger human class landers featuring multi-engine clusters at order of magnitude higher thrust levels than current robotic landers. Upward redirection of the plume flow and debris particles by the surface craters and damage of the landing surface topology pose landing risks that cannot be duplicated in terrestrial experiments or predicted with currently existing simulation tools.

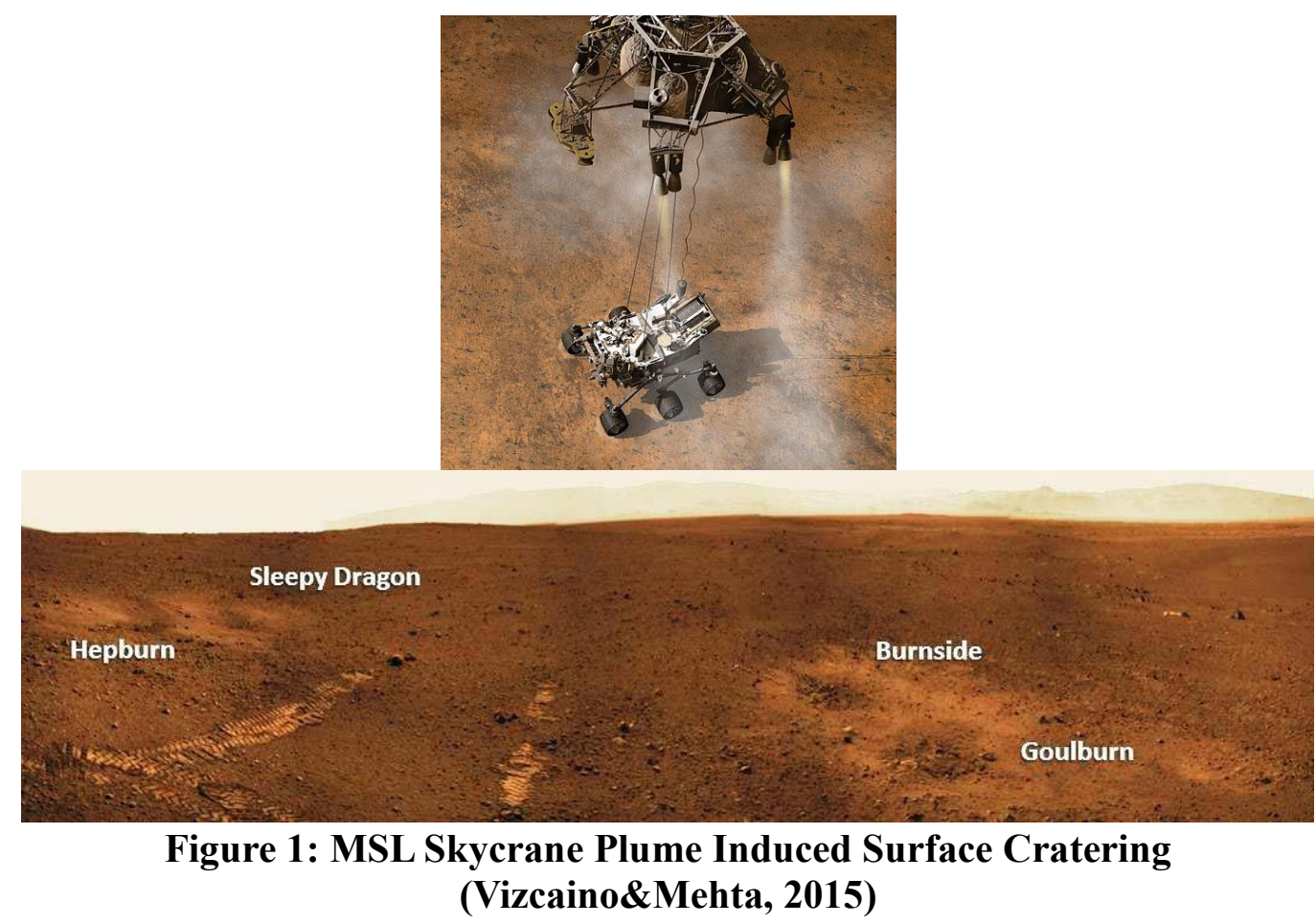

Numerical and experimental investigations of supersonic jet interactions with solid flat surfaces have been performed with traditional CFD programs in assessing the landing hazards of both the NASA Mars Science Laboratory and the Phoenix Mars spacecraft. (Mehta, 2013). Additionally, interactions of jet plumes with surface material simulant beds have been investigated in experimental tests to arrive at correlations for analyzing plume-soil erosion and prediction of erosion rates for those landers and future derived vehicles (Mehta, 2011). Such investigations have given useful insight into the erosion and cratering characteristics. However, no simulation framework existed that allows the analytical description of complex regolith granular materials and their response to an impinging jet flow. To fulfill this need, CFD Research Corporation (CFDRC) and the University of Florida have developed the Gas-Granular Flow Solver (GGFS) that solves the gas and granular phase interaction in a coupled manner with high fidelity granular phase particle physics modeling (Mehta, 2016) (Gale, 2017).

\section{GAS-GRANULAR FLOW SOLVER (GGFS)}


The regolith particle flow induced by a spacecraft landing occurs in a combination of "extreme environments" that combine low gravity, little or no atmosphere, with rocket exhaust gas flow that is supersonic and partially rarefied, and unusual mechanical properties of the regolith. Of these environmental factors, characterizing the regolith granular material fluidic behavior and gas-granular interactions is the most complex and least developed. The lunar regolith, as the extreme example, is poorly sorted with broad particle size distributions and large fines content, and gravel and cobbles embedded in the soil. It has significant cohesion, due to interlocking particle shapes for the very jagged particles. It is essential to develop the capability to model the response of such poly-disperse granular materials consisting of irregular shaped particles. The combination of particle shape and size distribution has been identified as major drivers in the complex particle flow response of the extra-terrestrial granular material.

Particle flow modeling approaches for both aspects, addressing the non-ideal particle shape and realistic particle distribution effects, have been developed within the last few years by the granular flow physics research community. CFDRC and the University of Florida implemented this technology to create the multi-phase, multi-physics GasGranular Flow Solver (GGFS) program. This development produced advanced computer modeling software with the unprecedented ability to predict the liberation and flow of realistic regolith granular material compositions in extra-terrestrial environments. Details of the gas-granular modeling approach and the development of the GGFS simulation tool can be found in (Gale, 2017). Only the salient features are summarized here.

The multi-phase GGFS features an Eulerian-Eulerian modeling approach, treating both the gas and granular material as continuum phases, to directly compute the interaction of the gas and granular phases at the surface and to directly simulate erosion, cratering and transport processes. The Lagrangian Discrete Element Method (DEM) offers the most detailed and accurate method of simulating the granular particle interactions and bulk flow behavior, and has been successfully applied for simple spherical as well as highly complex irregular particle shapes (Guo and Curtis 2015). However, the computational cost of DEM methods becomes prohibitive for granular flows on a landing site area where a simulation would have to track billions of particles individually. The Eulerian granular flow modeling approach allows simulation of granular flows with much lower computational cost when equipped with appropriate granular flow constituent models for inter-particle stress, viscosity, cohesion and kinetic energy across the range from densely packed to dilute particle phases. Our computational modeling makes use of both approaches; Lagrangian DEM and Eulerian continuum flow modeling. The DEM modeling is applied as a first principles method to extract and formulate the granular material physics constituent models. The granular material response closure models obtained from the small-scale detailed DEM simulations are then implemented in the Eulerian granular flow formulation. The Eulerian-Eulerian framework is then applied to efficiently simulate the gas-granular multi-phase flow. 
To enable the simulation of gas-solid flows with the Eulerian multi-phase solver, an Eulerian granular flow model has been implemented. Details of the computational model selected for our framework are described in an extensive review of granular physics models (Van Wachem\&Almstedt, 2003). The UF team developed a unique process to compose realistic and physically accurate granular flow constituent models (Guo\&Curtis, 2015). Inter-granular forces are dominated by the particle irregular shape and the resulting friction mechanisms affects properties of cohesion, compressibility, and shear strength. Constitutive models were formulated through first-principle 3-D DEM simulations for the non-spherical particles resulting in fundamental relationship between particle shape and particle-phase stress.

The 3-D governing equations for the multi-phase system consist of continuity, momentum and energy equations for each phase, as well as for the volume fraction of one of the phases. The granular mechanics model includes three components: 1) Collisional solid pressure and viscosity, 2) Kinetic solid pressure and viscosity and 3) Frictional solid pressure and viscosity. For the simulation of gas-solid systems a 'granular temperature' balance is employed. The granular temperature equation is solved to obtain the fluctuation energy of the solid phase. An open source Cartesian mesh flow solver framework with Adaptive Mesh and Algorithm Refinement (AMAR) capability is utilized to adapt to and resolve gas-solid interface physics in real time. The fully coupled AMAR capability provides significant increase in resolution and accuracy of the complex physics occurring between the phases.

The resulting software framework features the following essential components and simulation models:

- Non-staggered mesh pressure based flow solver.

- Multi-phase Eulerian phase formulation for gas and granular phase interaction.

- Compressible gas flow modeling, with gas phase turbulence modeling.

- Granular phase constituent models for idealized (spherical) and irregular particles.

- Constituent models for mono- and poly-disperse particle distribution.

- Granular temperature equation solver to obtain fluctuation energy of solid phase.

- Adaptive Cartesian mesh with automated mesh refinement and coarsening.

- Built upon an Open Source, object oriented, parallelized simulation framework.

The software development followed object oriented coding practices with rigorous version control. Software verification is performed with the Method of Manufactured Solutions (MMS) approach. Extensive validation of unit problems for single phase and multi-phase flow has been performed for free and impinging jets, dense granular material flow in hopper discharge, and dilute gas-granular flow for jet impingement on particle beds of regular and irregular granular materials such as Sand, glass beads, JSC$1 \mathrm{~A}, \mathrm{OB}-1$, and others. The simulation framework is now fully operational and has undergone application testing and technology demonstrations for propulsive lander applications. GGFS has been adopted by NASA organizations supporting propulsive landing concepts for human Mars lander concepts development and commercial lunar lander developments under the CATALYST program. 


\section{CURRENT SIMULATION CAPABILITIES FOR PLUME SURFACE INTERACTION AND RESULTING DEBRIS TRANSPORT}

The Fluid Dynamics Branch at the NASA Marshall Space Flight Center provides analytical and testing support in defining propulsion flow induced environments and has developed plume driven debris transport simulation capabilities for launch vehicle liftoff environments predictions. This debris transport analysis is achieved by combining the Loci/CHEM CFD tool for plume flow prediction and the NASA developed Debris Transport Analysis (DTA) post-processing toolkit for an integrated plume driven debris transport analysis capability. This capability has been in production use at NASA MSFC to support launch vehicle liftoff plume driven debris transport analysis for the Shuttle and Space Launch System (SLS) programs.

Loci/CHEM is the workhorse CFD analysis program used by the MSFC Fluid Dynamics Branch. The Loci framework (Luke, 1999) was developed with the goal of simplifying the development of complex numerical models that can take advantage of massively parallel high end computing systems. The Loci/CHEM flow solver (Luke, 2007) is a density-based Navier-Stokes solver implementation in the Loci framework for multi-component mixing and chemically reacting flows prevalent in launch vehicles and spacecraft propulsion flows. For complex geometry and object motion problems, the Loci/CHEM solver infrastructure supports overset meshes. Moving body simulations are performed with a 6-DOF model implementation capable of prescribed motion and unconstrained 6-DOF motion.

This DTA post-processing framework has been developed for Shuttle and SLS liftoff and ascent debris environment analysis [Gomez, 2004], [Williams, 2015]. It performs one-way coupled CFD-DTA post-processing of debris particle transport, only accounting for flow field effects on the particle trajectories but no feedback to the flowfield. The analysis begins with a CFD simulation of the plume induced flow field about the vehicle and impingement on the ground. The debris particle release locations are specified along with parametric variations in particle characteristics such as size, shape, density can be applied for multi-variate post-processing of the debris field. The DTA uses a debris tracing code to trace a debris particle's trajectory through the CFD flow field solution. The trajectory of the debris particle is determined by the aerodynamic forces exerted on the particle. An impact analysis program performs detection of whether the debris particle will impact objects in the domain, providing relative kinetic energy, velocity, and impact angle of the debris particle. Repeated postprocessing of the debris transport through the saved flow field with variations in particle sizes and other characteristics allows parametric assessment of the debris environment. The simulation is capable of performing transient debris transport simulations, tracing the debris trajectories through a time-varying flow solution such as the ascent of a launch vehicle or the propulsive descent of a lander.

The MSFC Fluid Dynamics Branch is currently applying the Loci/CHEM+DTA plume driven debris transport simulation framework in supporting the Mars Lander concept development efforts of the Mars Study Capability Team. Plume driven debris transport 
analysis is performed to provide insight into farfield plume driven surface regolith debris transport that defines the human Mars lander stay-out zone for landings in proximity to previously landed assets. Figure 2 shows an example of such a CFD-DTA analysis for an eight-engine Human Mars lander concept at an elevation of $10 \mathrm{~m}$ above the landing surface. The maximum extent of the debris particle trajectories for the full range of expected particle sizes defines the stay-out zone required for safe landing to protect existing surface infrastructure.

\begin{tabular}{l|l|l|}
\hline & \\
\hline
\end{tabular}

Figure 2. Example of Debris Transport Analysis Performed for Mars Human Lander Concept. Farfield Debris Transport Defines Safe Stay-Out Zone.

These simulations cannot account for the landing surface alteration through cratering induced by the plume impingement and the resulting flow and debris redirection. The newly adopted GGFS simulation capabilities have now be brought to bear for predicting the surface erosion and cratering topology.

One approach would be to integrate the GGFS plume-surface interaction and cratering prediction program into the existing plume-surface effects simulation and DTA prediction framework to replace the proven Loci/CHEM capabilities. The GGFS, however, does not currently offer several important features and capabilities provided by the Loci/CHEM tools. The GGFS simulation suite does not offer the ability to perform moving vehicle simulations to capture the effects of descent motion and resulting escalation of plume impingement effects. To capture the peak debris transport 
events requires a transient simulation of a moving spacecraft descent with simultaneous plume induced crater formation and evolution to capture the instance of maximum plume and debris stream upward redirection. It is not feasible to predict this occurrence from quasi-steady simulations at various frozen vehicle positions.

The Loci/CHEM suite provides such a very mature moving vehicle capability. Rather than integrate the GGFS capabilities into a LOCI program or integrate the moving body overset mesh capability into the GGFS framework, it was decided to apply both programs simultaneously, but for appropriate regions of the simulation domain. The effects of the descending vehicle are modeled with Loci/CHEM for the majority of the domain except for the region near the landing surface where the regolith cratering capabilities of GGFS required. The resulting patched but fully coupled simulation framework enabled the most efficient immediate application of both technologies to serve immediate simulation needs. A more complete full integration of both capabilities into a single simulation model will be undertaken in the future.

\section{INTEGRATED SIMULATION FRAMEWORK CHEM+GGFS+DTA}

This integrated multi-physics computational simulation framework has been implemented by the MSFC Fluid Dynamics Branch in cooperation with CFDRC to simulate the plume-surface interaction environment and resulting surface regolith layer erosions and cratering. The framework integrates the three simulation tools to achieve an end-to-end spacecraft landing plume-surface interaction dynamics prediction, plume induced surface cratering, and regolith particle debris transport towards the vehicle and surrounding assets. The components include the Loci/CHEM Computational Fluid Dynamics (CFD) simulation program, the Gas-Granular Flow Solver (GGFS), and the Debris Transport Analysis (DTA) toolkit.

The Gas-Granular Flow Solver (GGFS) simulation program is executed simultaneously with the Loci/CHEM CFD program in a tightly coupled simulation framework. Figure 3 depicts this simulation approach. The computational domain is subdivided into a farfield subdomain in which the plume flow field originating from the spacecraft is computed with the Loci/CHEM program and a near-surface subdomain in which the GGFS will be executed to predict the surface erosion and cratering effects. The two simulations communicate at an interface between the subdomains with full two-way coupling in space and time. For moving body simulations of a landing spacecraft, the overset mesh capability of the loci/CHEM framework is used with automatic interface updating with the GGFS region. The GGFS program and the CHEM/GGFS interfacing and communications functions have been configured as software modules in the Loci framework. The integrated simulation can therefore be performed with all the high-performance, massively parallel features of the Loci computational framework. The combined solution from the Loci/CHEM and the GGFS subdomains are saved in the Loci/CHEM output format for post-processing. The combined CHEM/GGFS computational solution output is then post-processed in the Debris Transport Analysis (DTA) framework to determine the transport of debris 
particles originating from the regolith surface. This integration of the three simulation tool components, Loci/CHEM, GGFS, and DTA, into a cooperative and coordinated simulation framework now enables high fidelity plume-induced surface cratering prediction and resulting regolith and rock debris transport and impact damage analysis for a realistic time-accurate descent and landing sequence.

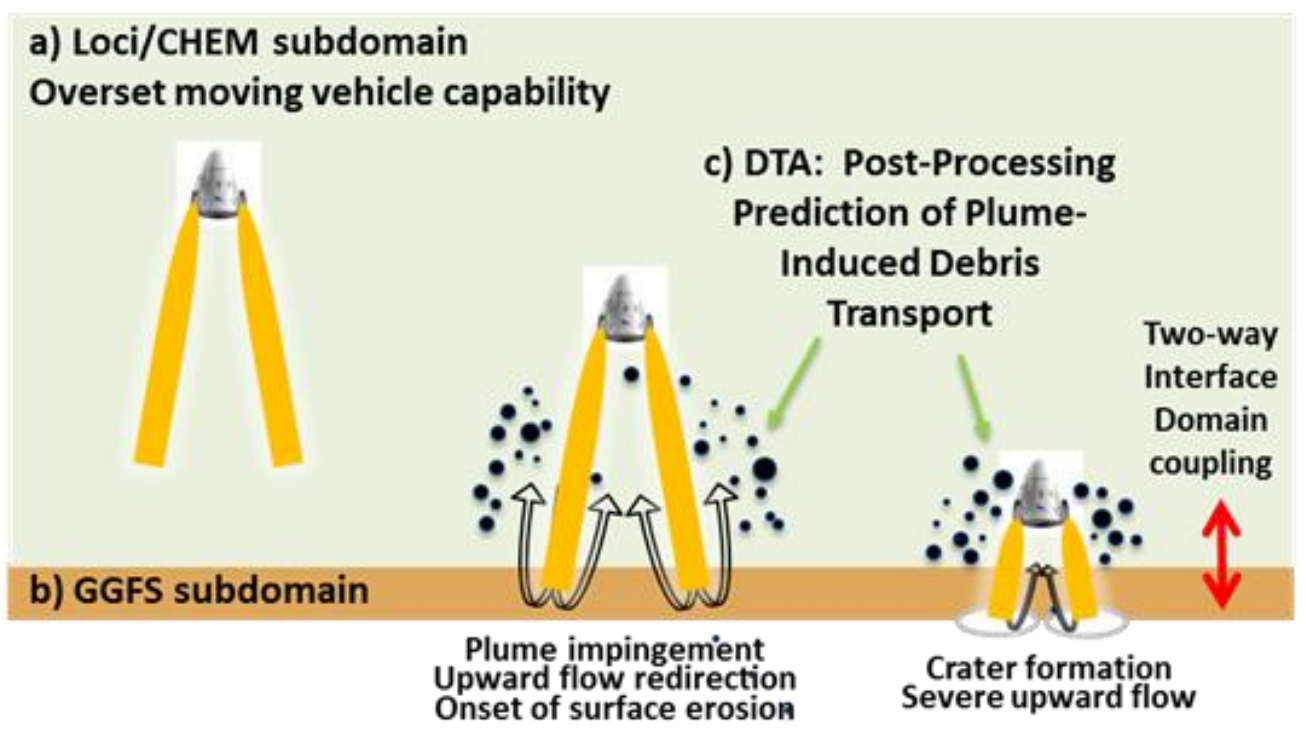

Figure 3. Plume-Surface Cratering Simulation Framework

\section{DEMONSTRATION OF LANDING SPACECRAFT PLUME-SURFACE CRATERING EFFECTS}

An axisymmetric proof of concept demonstration of all aspects of the simulation features the simulation framework was executed. The simulation modeled the plume impingement and crater formation of a notional spacecraft descending towards the landing surface composed of a bed of granular surface material. A sequence of still figures from this simulation is shown in Figure 4 . The granular material has a density of $2000 \mathrm{~kg} / \mathrm{m}^{3}$ with a particle size of 100 microns. The spacecraft is descending with constant vehicle downward velocity of $20 \mathrm{~m} / \mathrm{s}$. Initial height of vehicle is $28 \mathrm{~m}$ above the granular bed. Mach 3 nozzle flow exits from the nozzle exit with a one-meter diameter. The nozzle flow is impulsively started and the plume flow develops and impinges on the surface. The interface boundary between the Loci/CHEM and GGFS subdomains is indicated. This simulation thus demonstrated all aspects of a moving vehicle simulation with transient flow impingement and gas-granular surface crater formation.

The series of images in Figure 4 show the complex progression of the plume-induced surface cratering caused by a descending spacecraft:

(A) Propulsion activates on descending vehicle modeled with moving body CFD;

(B) Initial plume impingement and surface erosion; 
(C) Plume begins to erode a crater in the regolith surface;

(D) Plume direction is altered by growing crater and is directed upward toward the landing spacecraft.

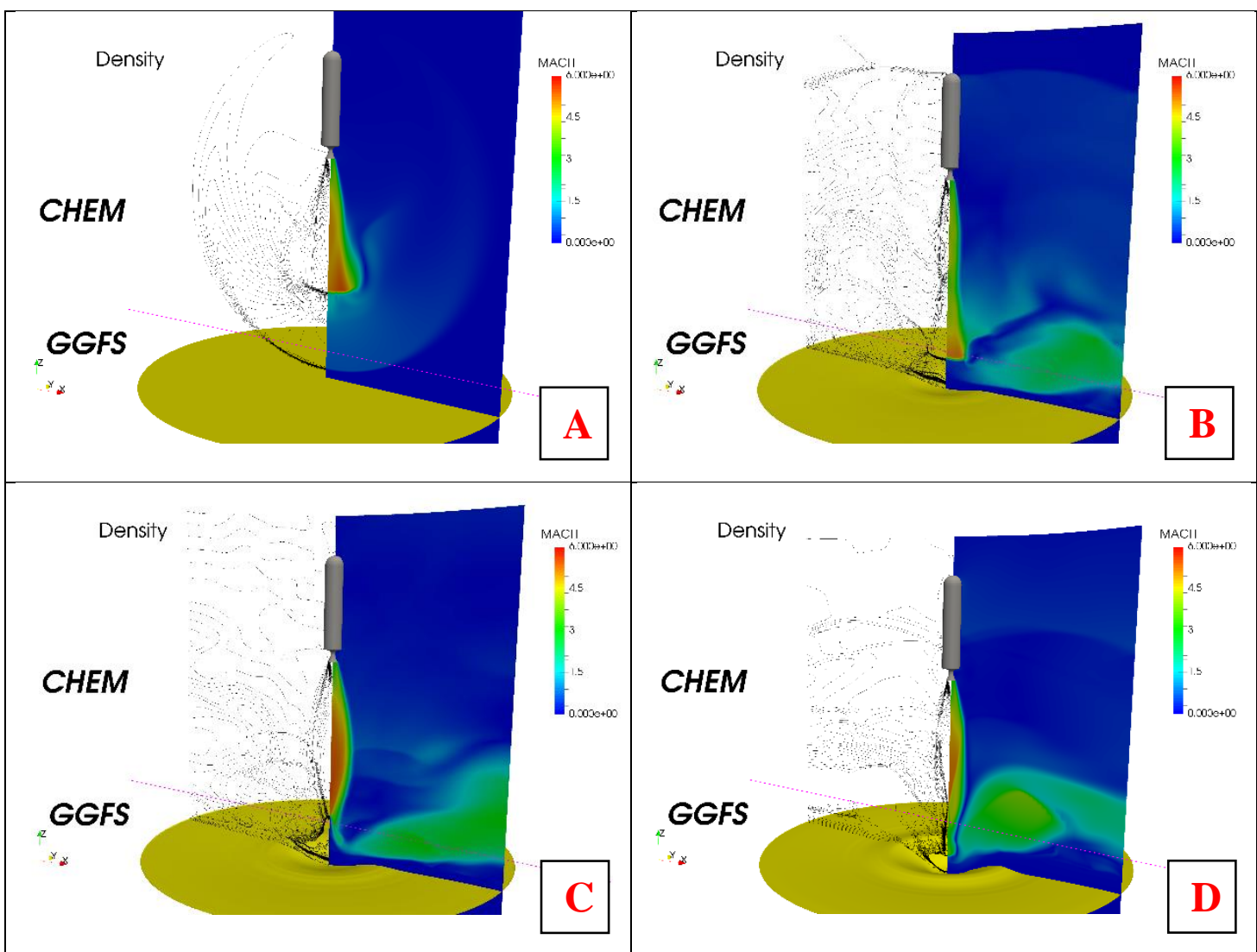

\section{Figure 4. Demonstration of Spacecraft Descent and Landing Plume Induced Surface Cratering}

This demonstration confirmed the feasibility of the coupled simulation framework for the complex plume induced crater formation process. The last component of the framework, the DTA analysis, can now be applied by releasing debris particles near the cratered surface and determining the debris trajectories. This allows identifying the effects of the surface topology alteration and the resulting plume flow redirection on the debris environment.

\section{FUTURE WORK}

The integrated simulation framework has been fully implemented on NASA supercomputers and demonstrated in the 2-dimensional simulation presented above. All computational and modeling aspects of the simulation framework have thus been proven operational. All simulation components are fully capable of two- and threedimensional simulations. The next step is to scale up the simulation capability to perform three-dimensional multi-plume cratering simulations and establish production readiness to support NASA projects. The Loci/CHEM CFD simulations capabilities for complex three-dimensional full vehicle liftoff and landing simulations have been 
proven in every day NASA applications. Focus is now on the scaling of GGFS simulations in the supercomputer environment for multi-plume impingements on landing site size granular surface beds.

\section{CONCLUSION}

The NASA Marshall Space Flight Center Fluid Dynamics Branch and CFDRC are currently integrating the Gas-Granular Flow Solver (GGFS) capabilities into a proven NASA plume-surface interaction and debris transport simulation framework featuring the Loci/CHEM CFD program and NASA developed Debris Transport Analysis (DTA) post-processing tools for applications in robotic and human lander development. The framework integrates the three simulation tools to achieve an end-to-end spacecraft landing plume-surface interaction dynamics prediction, plume induced surface cratering, and regolith particle debris transport towards the vehicle and surrounding assets. The soundness of this approach was demonstrated with an axisymmetric timeaccurate simulation of the plume impingement and surface cratering for a landing spacecraft. The simulation confirmed the complex two-way effects of the surface alteration by the plume flow and the reciprocal alteration and redirection of the plume flow with strong influence on the resulting debris particle transport environment. The framework is now being matured to enable efficient three-dimensional, multi-engine plume impingement cratering simulations for realistic lander concepts.

\section{REFERENCES}

Gale, M. P., Mehta, R. S., Liever, P. A., Buettner, K. E., and Curtis, J. S., "GasGranular Flow Solver for Plume Surface Interaction and Cratering Simulations." AIAA Paper 2017-4503.

Gomez, R. J., Vicker, D., Rogers, S. E., Aftosmis, M. J., Chan, W. M., Meakin, R., and Murman, S., "STS-107 Investigation Ascent CFD Support," AIAA Paper 20042226.

Guo, Y. and J. S. Curtis (2015). "Discrete element method simulations for complex granular flows." Annual Review of Fluid Mechanics 47: 21-46.

Luke, E. A., "A Rule-Based Specification System for Computational Fluid Dynamics," PhD Thesis, Mississippi State University, Mississippi, 1999.

Luke, E. A., "On robust and accurate arbitrary polytope CFD solvers," AIAA Paper 2007-3956.

Mehta, M., Renno, N. O., Marshall, J., Grover, M. R., Sengupta, A., Rusche, N. A., Kok, J. F., Arvidson, R. E., Markiewicz,W. J., Lemmon, M. T., and Smith, P. H., "Explosive Erosion During the Phoenix Landing Exposes Subsurface Water on Mars," Icarus, Vol. 211, No. 1, 2011, pp. 172-194.

Mehta, M., Sengupta, A., Renno, N. O., Van Norman, J. W., Huseman, P. G., Gulick, D. S., Mark Pokora, M., "Thruster Plume Surface Interactions: Applications for Spacecraft Landings on Planetary Bodies." AIAA Journal, Vol. 51, No. 12, Dec. 2013. 
Mehta, R. S., Liever, P. A., Salmon, J. L., Buettner, K. E., and Curtis, J. S., "Particle Flow Physics Modeling for Extreme Environments." Earth and Space 2016: 131-141.

Van Wachem, B. and Almstedt, A.-E., "Methods for multiphase computational fluid dynamics." Chemical Engineering Journal 96(1): 81-98, 2003.

Vizcaino, J. and Mehta, M., "Quantification of plume-soil interaction and excavation due to the sky crane descent stage." AIAA Paper 2015-1649.

Williams, B., "Analyzing the Space Launch System Debris Environment," https://www.nas.nasa.gov/SC15/demos/demo13.html , SC15 Supercomputing Conference, 2015. 